\title{
Comparação dos métodos tradicional e ativo de educação no aprendizado de um tema de Oclusão Dentária
}

Fernanda Nunes de Souza*; Renata Nunes Barros**; Cristina Costa de Almeida***; Marceli de França****; Armando Hayassy*****

\section{RESUMO}

Este trabalho avalia o efeito dos métodos de ensino tradicional e ativo aplicados ao conteúdo "montagem de modelos em articulador semiajustável (ASA)". Trata-se de um estudo transversal em relatórios de 49 alunos da disciplina de Oclusão, divididos aleatoriamente em dois grupos de atendimento mútuo em duplas. O grupo $1(n=26)$ recebeu um artigo sobre o tema e as dúvidas foram resolvidas em debate, o grupo $2(n=23)$ participou de aula expositiva seguida das práticas. Os dados foram compilados quanto às taxas de acertos nas etapas arco facial, montagem do modelo superior, registro intermaxilar, compensação da cera e montagem do modelo inferior. Foi realizada uma demonstração prévia de cada etapa e permitidos 20 minutos para realizar a atividade. Posteriormente, o grau de dificuldade foi quantificado. Foram considerados corretos os casos que reproduziram os contatos do paciente nos modelos. No Grupo $126,1 \%$ dos alunos apresentaram correta montagem e no Grupo 2 nenhum aluno apresentou correta montagem, $24,5 \%$ dos erros ocorreram na fase compensação de cera, $36,7 \%$ no registro intermaxilar, $2 \%$ na montagem do modelo inferior e nenhum na fase do arco facial. Os
Professora nas disciplinas de oclusão e prótese dentária na faculdade de Odontologia das Faculdades São José, Rio de Janeiro Doutora em Odontologia UFF, Niterói, RJ

Professora Adjunta UFF, Niterói, RJ

Especialista em Prótese Dentária

Diretor da Faculdade de Odontologia das Faculdades São José, Rio de Janeiro, RJ

resultados sugerem que as técnicas ativas de aprendizado influenciaram positivamente e confirmam a necessidade de material didático específico para o entendimento e reprodução das relações intermaxilares, dirigido aos alunos em transição do ciclo teórico para o ciclo prático.

Descritores: Educação Superior. Educação em Odontologia. Aprendizagem. Oclusão Dentária.

\section{INTRODUÇÃO}

Apesar da crescente preocupação dos órgãos de Saúde Pública com a saúde bucal e de estudos que comprovam a redução dos índices de perda dental no Brasil, é grande o número de pessoas parcial ou totalmente edentadas que necessitam de reabilitação bucal ${ }^{1-5}$. No Brasil, o Ministério da Educação recomenda que os cursos de Odontologia formem profissionais generalistas capazes de atender às necessidades de saúde bucal da comunidade ${ }^{6}$.

O conhecimento sobre reabilitação bucal deve ser adquirido pelo estudante de Odontologia durante a graduação ${ }^{6}$. Os conceitos de oclusão norteiam o planejamento e a execução da reabilitação, marcando a • 15(4):60-66, 2015. 
transição do ciclo básico do ensino de Odontologia para o ciclo profissional, que é direcionado à prática clínica ${ }^{7,8}$.

Os métodos de avaliação da acurácia de procedimentos desenvolvidos pelos alunos de graduação constituem um recurso útil para a saúde daqueles que dependem desse serviço.

O ensino da Odontologia deve ter como orientação a adequação às necessidades e expectativas dos pacientes, proporcionando aos alunos o aprendizado voltado para a qualidade do trabalho reabilitador a ser realizado, com o correto planejamento, permitindo ótimo desempenho biomecânico e respeitando os princípios básicos de oclusão9 9 . Desta forma, as Instituições de Ensino Superior têm, entre suas atribuições, preparar e conscientizar os docentes quanto à responsabilidade de ensinar o conteúdo teórico e cultivar nos discentes o desenvolvimento da reflexão sobre as implicações dos resultados de pesquisas científicas na prática odontológica ${ }^{7,10,11}$.

Além disso, universitários inscritos em um projeto de reorientação profissional de uma instituição pública, demonstraram insatisfação com as práticas educativas tradicionais e, em especial com o distanciamento entre a teoria e a prática $^{12}$. Tal aspecto é frequentemente apontado como principal em estudos que buscaram entender os motivos relacionados à evasão ${ }^{13-16}$ e escolha profissional ${ }^{17-20}$.

Neste contexto, o presente trabalho objetiva avaliar o desempenho dos alunos da disciplina de oclusão da Faculdade de Odontologia das Faculdades São José, Rio de Janeiro/Brasil, comparando o efeito da aula expositiva ao da leitura e debate um artigo, na aplicação dos conceitos teóricos na montagem de modelos de estudo em articulador semiajustável (ASA), bem como na aplicação dos conceitos de oclusão na análise dos mesmos.

\section{MATERIAL E MÉTODOS}

A pesquisa foi aprovada pelo comitê de ética institucional (processo 035/09). Dos 55 estudantes do terceiro período do curso de graduação em Odontologia das Faculdades São José (Rio de Janeiro, Brasil) inscritos na disciplina de Oclusão no segundo semestre de 2014, 49 concordaram em participar da pesquisa e firmaram o Termo de Consentimento Livre e Esclarecido. Destes, 26 eram do turno da manhã (Grupo 1) e 23 no turno da tarde (Grupo 2). A idade média foi de $25 \pm 6$ anos, variando de 19 a 46 anos. A metodologia consistiu de três etapas, a seguir descritas.

\section{Conceitos}

$\mathrm{Na}$ primeira etapa os alunos receberam informações sobre anatomia, princípios básicos de oclusão, métodos de exame clínico e diagnóstico. Estes conceitos foram abordados sob a forma de leitura e debate de artigo científico sobre o tema ${ }^{21}$ (método ativo) com o Grupo 1 e em aulas teóricas (método tradicional) com o Grupo 2.

\section{Montagem dos modelos em ASA}

$\mathrm{Na}$ segunda etapa os participantes de ambos os grupos receberam a demonstração de todas as etapas da atividade a ser desenvolvida e trabalharam em duplas de atendimento mútuo, com tempo de 20 minutos para a montagem dos modelos em ASA.

Esta atividade prática se deu na clínica para o Grupo 1 e em laboratório para o Grupo 2. Todos os alunos foram avaliados em relação às etapas de trabalho: montagem do arco facial, montagem do modelo superior no arco facial, registro intermaxilar em cera, compensação da cera e montagem do modelo inferior. Em cada etapa de trabalho os alunos recebiam uma nota considerando o número de repetições necessárias para alcançar o objetivo e o número de solicitações de ajuda.

\section{Conferência dos contatos oclusais e preenchimento de relatório}

Nesta etapa foram, inicialmente, comparados os contatos obtidos no modelo de Revista da ABENO • 15(4):60-66, 2015. 
gesso com aqueles encontrados na boca, utilizando papel carbono. Foi considerada montagem correta quando houve coincidência entre os contatos oclusais no aluno tido como "paciente" e os modelos montados em ASA.

A seguir, os alunos responderam sobre seu grau de dificuldade na realização de cada etapa e o número de auxílios solicitados.

Os dados dos relatórios foram tabulados de forma a permitir a avaliação da porcentagem de erros e acertos em cada etapa.

\section{RESULTADOS}

Para a maioria dos alunos (46-93,8\%) este foi o primeiro contato com o procedimento proposto, os demais já haviam realizado cursos técnicos na área, como, técnico em prótese dentária e em higiene bucal ou tiveram experiência em clínicas ou consultórios odontológicos.

Entre os participantes do estudo 35 $(71,4 \%)$ eram mulheres e $14(28,6 \%)$ homens. Na tabela 1 são descritos os erros por etapa e grupo, assim como por gênero. Observou-se que no grupo 1, $6(26,1 \%)$ alunos realizaram correta montagem dos modelos e no 2 nenhum aluno apresentou este resultado. Os erros mais prevalentes em ambos os grupos ocorreram na fase compensação de cera 12 (24,5\%), no registro intermaxilar, $18(36,7 \%)$ na montagem do modelo inferior e $1(2 \%)$ na fase do arco facial (0).

Além disso, 12 alunos (24,5\%), não entregaram o relatório das atividades. Tal fato indica ausência no último dia das atividades práticas e, portanto, a não realização da checagem dos registros, sendo considerada montagem incompleta.

Tabela 1 - Erros cometidos pelos integrantes dos grupos em estudo, por etapa da atividade e por gênero.

\begin{tabular}{c|c|c|c|c|c|c|c|c}
\hline \multirow{2}{*}{ Grupo } & \multirow{2}{*}{ Gênero } & $\begin{array}{c}\text { Arco } \\
\text { facial } \\
\mathrm{n}-\%\end{array}$ & $\begin{array}{c}\text { Modelo } \\
\text { Superior } \\
\mathrm{n}-\%\end{array}$ & $\begin{array}{c}\text { Registro } \\
\text { Intermaxilar } \\
\mathrm{n}-\%\end{array}$ & $\begin{array}{c}\text { Compensação } \\
\text { Cera } \\
\mathrm{n}-\%\end{array}$ & $\begin{array}{c}\text { Modelo } \\
\text { Inferior } \\
\mathrm{n}-\%\end{array}$ & $\begin{array}{c}\text { Montagem } \\
\text { Correta } \\
\mathrm{n}-\%\end{array}$ & $\begin{array}{c}\text { Sem } \\
\text { Relatório } \\
\mathrm{n}-\%\end{array}$ \\
\hline \multirow{2}{*}{1} & Feminino & $0-0$ & $0-0$ & $7-38,8$ & $7-38,9$ & $0-0$ & $2-11,1$ & $4-22,2$ \\
& Masculino & $0-0$ & $0-0$ & $2-25,0$ & $1-12,5$ & $0-0$ & $4-50,0$ & $1-12,5$ \\
& Total & $0-0$ & $0-0$ & $9-34,6$ & $8-30,8$ & $0-0$ & $6-23,0$ & $5-19,2$ \\
\hline \multirow{2}{*}{2} & Feminino & $0-0$ & $0-0$ & $2-10,5$ & $7-41,2$ & $1-5,3$ & $0-0$ & $3-17,6$ \\
& Masculino & $0-0$ & $0-0$ & $1-16,6$ & $3-37,5$ & $0-0$ & $0-0$ & $4-66,6$ \\
& Total & $0-0$ & $0-0$ & $3-13,0$ & $10-43,5$ & $1-4,3$ & $0-0$ & $7-30,4$ \\
\hline
\end{tabular}

\section{DISCUSSÃO}

$\mathrm{O}$ presente trabalho avaliou $\mathrm{O}$ desempenho dos alunos do terceiro período da graduação de Odontologia na aplicação dos conceitos teóricos na montagem de modelos de estudo em ASA, bem como na aplicação dos conceitos de oclusão na análise dos mesmos.

De acordo com os resultados houve maior número de mulheres $(70 \%)$ que de homens (30\%). Alguns estudos demonstrem tendência similar ${ }^{1-3,5}$, com maioria feminina em pesquisas. Pellizzer $(2013)^{2}$ afirma que este resultado ocorre porque no Brasil há um maior número de mulheres que homens.

Quanto à diferença entre os gêneros no desempenho na montagem do ASA, este estudo demonstrou proporção de montagem correta de $50 \%$ para homens e $11 \%$ para mulheres, não 
havendo equilíbrio entre os gêneros no grupo 1 . No grupo 2 não houve nenhuma montagem correta. Este resultado contradiz alguns relatos, pois quanto às diferenças de gênero em relação ao estudo e dedicação às atividades exploratórias, gênero feminino apresenta maior nível de comportamento exploratório do que o masculino, com diferenças significativas nas dimensões de busca interna, busca externa e intencionalidade, sugerindo que as mulheres tendem a se engajar de forma mais sistemática e deliberada em atividades exploratórias ${ }^{22}$. No entanto, a literatura não é consistente em relação à diferença de gênero na exploração; enquanto alguns estudos confirmam a maior exploração feminina ${ }^{23}$, outros indicam o contrário $^{24}$ ou não apontam diferenças ${ }^{25,26}$. Os estudos que indicam maior exploração masculina tendem a justificá-la pela maior percepção de oportunidades de trabalho e menor percepção de barreiras por parte dos homens.

Estudos realizados com universitários mostram que ser capaz de tomar decisões eficientes está relacionado à busca sistemática de informações sobre as próprias habilidades e interesses e sobre oportunidades educacionais e ocupacionais. Em uma pesquisa com recém graduados, Werbel $(2000)^{27}$ observou que a capacidade exploratória é um importante passo inicial para a busca de emprego; o autor afirma que os processos de aconselhamento de carreira devem priorizar o desenvolvimento dessa capacidade. Texeira $(2002)^{28}$ identificou relações entre maior comportamento exploratório e otimismo para a busca de emprego entre formandos universitários.

Entretanto quanto à aplicação dos conceitos teóricos, Neto et al. (2010) ${ }^{6}$ realizaram uma avaliação do conhecimento de formandos em Odontologia sobre o planejamento e preparo da boca para a realização de prótese parcial removível, através do exame de duas armações metálicas montadas em ASA. Com uma amostra composta por 266 alunos (23\%) de Odontologia do estado de São Paulo, de 11 diferentes cursos, concluíram não existir conhecimento adequado sobre o assunto. Os autores relataram não haver correlação estabelecida entre as instruções didáticas e clínicas oferecidas na graduação para a confecção de próteses parciais removíveis e o comportamento inapropriado dos estudantes avaliados. Duas hipóteses foram apresentadas como capazes de explicar este fato: os graduandos negligenciam o conhecimento adquirido sobre o assunto para ganhar tempo ou os mesmos recebem instruções inadequadas durante a graduação.

Marshall et al. (2014) ${ }^{11}$ estudaram as escolhas dos estudantes de Odontologia entre artigos e métodos de pesquisa, assim como sua habilidade de refletir sobre as implicações de seus resultados para a prática odontológica. Este estudo mostrou a necessidade de critérios para a avaliação dos fatores que levam os estudantes a refletir sobre a ligação entre a ciência e o trabalho clínico, além de motivá-los a aprender a refletir para se tornarem pensadores críticos. Sugerem ainda que os estudantes de Odontologia deveriam aprender mais sobre métodos qualitativos de pesquisa.

Quanto à relação experiência e prática clínica, Super $(1955)^{19}$ criou o termo maturidade em um período inicial de suas formulações teóricas, para avaliar o estágio de desenvolvimento de carreira alcançado por estudantes de diferentes idades e níveis de instrução e sua respectiva prontidão para a tomada de decisões. Com o passar do tempo, segundo Magalhães $(2005)^{16}$, ao descrever a carreira na vida adulta e perceber que as habilidades, competências e atitudes de enfrentamento podem não variar com a idade, Super $(1983)^{20}$ passou a utilizar o termo adaptabilidade de carreira, indicando equilíbrio entre o mundo do trabalho e o espaço pessoal, além de habilidades para buscar e aceitar 15(4):60-66, 2015. 
mudanças nos papéis de carreira ao longo do tempo.

As dimensões da maturidade propostas por Super $(1983)^{20}$ seriam: a) capacidade de planejamento, que depende da autonomia do indivíduo, da adoção de uma perspectiva temporal e da auto avaliação das condições favoráveis ou desfavoráveis em relação à carreira, estando relacionada também à autoestima; b) capacidade de exploração, com presença ou não do comportamento exploratório vocacional, o qual permite diferenciar entre escolhas racionais e refletidas daquelas impulsivas ou dependentes; c) informação, pois a informação sobre o mundo do trabalho e as opções oferecidas é um prérequisito para a prontidão para a tomada de decisão; d) tomada de decisão, habilidade decorrente da avaliação das possibilidades, das consequências possíveis destas decisões e da probabilidade destas consequências acontecerem; e e) orientação à realidade, que consiste em autoconhecimento, realismo e avaliação situacional. O conjunto destas características compõe a prontidão para a tomada de decisão ou, mais tecnicamente, a prontidão do indivíduo para emitir os comportamentos necessários em resposta às demandas sociais de cristalização e especificação de escolhas e/ou mudanças vocacionais $^{18}$.

Valore et al. (2010) $)^{12}$ investigaram os fatores relacionados à dúvida/ insatisfação quanto ao curso escolhido no discurso de universitários de uma instituição pública, inscritos num projeto de reorientação profissional e evidenciaram a insatisfação dos alunos avaliados com as práticas educativas, em especial com o distanciamento entre a teoria e a prática. Tal aspecto é frequentemente apontado, como fator principal, em estudos que buscaram entender os motivos relacionados à evasão ${ }^{13-16}$.

Torna-se notável uma preocupação excessiva com avaliação ao invés da valorização da aquisição do conhecimento e do domínio da técnica. Os alunos, por razões variadas como falta de tempo, desconhecimento de oportunidades ou mesmo desinteresse, podem estar perdendo chances importantes de treinar as habilidades e competências que serão solicitadas na atividade profissional ${ }^{17}$.

A proposta curricular atual sugere a quebra do modelo de ensino baseado na perspectiva bancária de acúmulo e transmissão do saber $^{29}$ e do modelo flexneriano ${ }^{30}$ e implantação de um ensino voltado para a autoaprendizagem, aprender a aprender e busca autônoma do conhecimento. Vê-se um processo baseado no desenvolvimento de competências, ou seja, na capacidade do profissional/estudante mobilizar conhecimentos em diferentes áreas do conhecimento, para a resolução dos problemas concretos encontrados nas atividades diárias $^{31,32}$.

\section{CONCLUSÃO}

Os resultados do presente estudo sugerem que a técnica ativa de aprendizado associada à prática em clínica odontológica influenciaram positivamente nos resultados, ao passo em que as atividades tradicionais em laboratório com aulas expositivas não apresentaram resultados satisfatórios. Os resultados confirmam a necessidade da criação de material didático específico para o entendimento e reprodução das relações intermaxilares, dirigido aos alunos em transição do ciclo teórico para o ciclo prático. Contudo, é necessário despertar o interesse do aluno para o pensar, questionar, aprender, fazer e assumir uma responsabilidade profissional.

\section{ABSTRACT \\ Comparison of traditional education and active learning methods in a subject of dental occlusion \\ This study evaluates the effects of traditional} Revista da ABENO • 15(4):60-66, 2015. 
and active teaching methods applied in the assembly of models in Semi-adjustable articulator (ASA). It is a cross-sectional study of 49 students reports Occlusion discipline, randomly divided into two groups of mutual care in pairs. Group $1(\mathrm{n}=26)$ received an article on the subject and doubts resolved in debate, group $2(n=23)$ participated in lecture followed by practice. The data were compiled as the hit rates in steps: facial arc, mounting the upper model, intermaxillary record, compensation wax, mounting the lower model. At each stage a demonstration was held 20 minutes to perform the activity and quantified the degree of difficulty. The cases were considered correct analyzing the touches in the models and in the patient. The morning shift, $23 \%$ of students present correct assembly and in the afternoon no students presented correct assembly, $42.9 \%$ of the errors occurred in the compensation phase of wax, $24.5 \%$ in intermaxillary record, $14.3 \%$ in the assembly the lower model and $6 \%$ at the stage of facial arc. The results suggest that active learning techniques positively influenced and confirm the need for specific educational material for understanding and reproduction of intermaxillary relations, aimed at students in transition from theoretical to practical cycle.

Descriptors: Education, Higher. Education, Dental. Learning. Dental Occlusion.

\section{REFERÊNCIAS}

1. Charyeva OO, Altynbekov KD, Nysanova BZ. Kennedy classification and treatment options: A study of partially edentulous patients being treated in a specialized prosthetic clinic. J Prosthodont 2012; 21(3):177-80.

2. Pellizzer EP, Almeida DA, FalcónAntenucci RM, Sánchez DM, Zuim PR, Verri FR. Prevalence of removable partial dentures users treated at the Aracatuba Dental School-UNESP. J Prosthodont 2013; 22(3):245-9.

3. Perera R, Ekanayake L. Tooth loss in Sri Lankan adults. Int Dent J 2011;61:7-11.

4. Souza FN, De Siqueira GC, Rodrigues ARC, Tiossi R, De Gouvea, Cresus VD, De Almeida CC . Partially edentulous arches: a 5 year survey of patients treated at the Fluminense Federal University Removable Prosthodontics Clinics in Brazil. J Prosthodont 2015;24(6):447-51.

5. Teófilo LT, Leles CR. Patients' selfperceived impacts and prosthodontic needs at the time and after tooth loss. Braz Dent $\mathbf{J}$ 2007;18(2):91-6.

6. Neto A F, Duarte ARC, Shiratori FK, Leite PHAS, Rizzatti-Barbosa CM.; Bonachela WC. Evaluation of senior Brazilian dental students about mouth preparation and removable partial denture design. J Dent Educ 2010; 74(11):1255-60.

7. Carcereri DL, Padilha ACL, Bastos RC. Fatores relevantes para a mudança na formação em Odontologia motivados pelo Programa Nacional de Reorientação da Formação Profissional em Saúde (PróSaúde). Rev ABENO 2014;14(1):94-106.

8. McNeil C. Science and Practice of Occlusion. $1^{\mathrm{a}}$ ed. Chicago: Editora Quintessence Publish. Co. Inc.; 1997.

9. Souza, FN, Gouvea CVD, Costa YO, Rodrigues ARC, Campos ED, Almeida CC. Análise in vitro da adaptação de apoios em prótese parcial removível. Rev Flum de Odontol 2011; 36: 10-7.

10. Hendricson WD, Anderson E, Andrieu SC, Chadwick DG, Cole J R, George MC, et al. Does faculty development enhance teaching effectiveness? J Dent Educ 2007; 71(12):1513-33.

11. Marshall TA, Straub-Morarend CL, Handoo N, Solow CM, Cunningham-Ford MA, Finkelstein MW. Integrating critical thinking and Evidence-Based Dentristy across a four-year Dental Curriculum: a model for independent learning. J Dent Educ 2014;78(3):359-67.

12. Valore LA, Ferrarini N L. Escolha e identidade profissional: desafios e possibilidades na formação universitária. Rev INFAD de Psicologia 2010;03:39198.

13. Biazus C. Sistema de fatores que levam o aluno a evadir-se dos cursos de graduação na UFSM e na UFSC: um estudo no curso de ciências contábeis. [Tese] Florianópolis (SC): Universidade Federal de Santa 
Catarina; 2004

14. Cunha AM, Tunes E, Silva RR. Evasão do curso de química da Universidade de Brasília: a interpretação do aluno evadido. Quím Nova 2001;24(2):262-80.

15. Ghizoni LD, Teles MMR. Escolha e reescolha profissional: um estudo sobre estudantes universitários noturnos. In: Lassance, MCP (Org.) Intervenção e compromisso social: orientação profissional. São Paulo: Vetor; 2005. p. 291-9.

16. Magalhães M, Redivo A. Re-opção de curso e maturidade vocacional. Rev Bras Orientac Prof 1998;2(2):7-28.

17. Bardagi MP, Boff RM. Autoconceito, auto eficácia profissional e comportamento exploratório em universitários concluintes. Avaliação 2010;15(1):41-56.

18. Savickas ML. Measuring career development: current status and future directions. Career Dev Q 1994;43:54-61.

19. Super DE. The dimensions and measurement of vocational maturity. Teach Coll Rec 1955;57:151-63.

20. Super, DE. Assessment in career guidance: Toward truly developmental counseling. Pers Guid J 1983; 61: 555-62.

21. Fernandes Neto, AJ et al. Montagem de modelos de estudo em articulador classe III semi-ajustável - ASA. 2005. Disponível em: http://www.fo.ufu.br/sites/fo.ufu. br/files/Anexos/Documentos/Anexos RoteiroOclusaoCap13.pdf

22. Frischenbruder SL. O desenvolvimento vocacional na adolescência: Autoconceito e comportamento exploratório. [Tese] Porto Alegre (RS): Universidade Católica do Rio Grande do Sul; 1999.

23. Sparta M. A exploração e a indecisão vocacional em adolescentes no contexto educacional brasileiro. [Tese] Porto Alegre (RS): Universidade Federal do Rio Grande do Sul; 2003.
24. Blustein DL, Pauling ML, De Mania ME, Faye M. Relation between exploratory and choice factors and decisional process. J Vocat Behav 1994;44(1):75-90.

25. Ketterson TU, Blustein DL. Attachment relationships and the career exploration process. Career Dev Q 1997;46:167-78.

26. Sparta M, Bardagi MP, Andrade AMJ. Exploração vocacional e informação profissional percebida em estudantes carentes. Aletheia 2005;22:79-88.

27. Werbel J D. Relationships among career exploration, hob search intensity, and job search effectiveness in graduating college students. J Vocat Behav 2000;57:379-94.

28. Teixeira MAP. A experiência de transição entre a universidade e o mercado de trabalho na adultez jovem. [Tese] Porto Alegre (RS): Universidade Federal do Rio Grande do Sul; 2002.

29. Freire P. Pedagogia do Oprimido. $1^{\mathrm{a}}$ ed. Rio de Janeiro: Editora Paz e Terra; 1970.

30. Almeida Filho, N. Reconhecer Flexner: inquérito sobre produção de mitos na educação médica no Brasil contemporâneo. Cad Saúde Pública 2010;26(12): 2234-49.

31. Lemos SGL, Morita M C, Carloto EET. Recursos utilizados por graduandos de odontologia e cirurgiões-dentistas do SUS para estudar e resolver dúvidas surgidas durante o trabalho. Rev ABENO 2014; 14(1): 3-16.

32. Perrenoud P. Construir as competências desde a escola. $1^{\text {a }}$ ed. Porto Alegre: Artmed; 1999.

Correspondência para:

Prof. Armando Hayassy

e-mail: ahayassy@uol.com.br

Rua Augusto da Cruz Nunes, 59 sobrado,

Pendotiba/Badu

24320-140 Niterói - RJ 